\title{
Cooperative atomic scattering of light from a laser with a colored noise spectrum
}

\author{
Xiaoji Zhou* \\ School of Electronics Engineering \& Computer Science, Peking University, Beijing 100871, China
}

(Dated: November 12, 2018)

\begin{abstract}
The collective atomic recoil lasing is studied for an ultra-cold and collisionless atomic gas in a partially coherent pump with a colored noise. Compared to white noise, correlations in colored noise are found to be able to greatly enhance or suppress the growth rate, above or below a critical detuning. Effects on cooperative scattering of light for noise correlation time, noise intensity and pump-probe detuning are discussed. This result is consistent with our simulation and linear analysis about the evolution equations in the regions of instability.
\end{abstract}

PACS numbers: 42.60.Mi,05.45.Xt, 42.65.Sf, 42.50.Wk

\section{INTRODUCTION}

Collective nonlinear interactions between cold atoms and light have attracted considerable attention since collective atomic recoil lasing (CARL) was observed in cold atoms [1, 2] and superradiance from Bose-Einstein Condensation (BEC) was realized in experiments [3]. The signature of CARL is sudden buildup in the probe field or back-scattering beam oriented reversely to a coherent pump strongly interacting with an atomic gas [4, 5, 6]. This self-organization phenomenon shows spontaneous formation of an atomic density grating, and offers the possibility to study the light amplification derived from collective interaction of light with cold atomic gases [7, 8, 9, 10].

Most studies so far have focused on the coherent laser pump, neglecting the fact that environment is intrinsically noisy. As found in a recent work for a partially coherent pump field with a white noise spectrum [11], the probe intensity can be larger than that in a coherent pump due to the noise. The intensity fluctuation and noise play important roles in this nonlocally-coupled many-body system. Although the white-noise assumption is convenient for mathematical treatment, it is somewhat unrealistic, because fluctuations in the microscopic dynamics have a finite (nonzero) correlation time, commonly referred to as colored noise [12]. The white noise approximation is valid when the correlation time of fluctuation is much shorter than all other relevant time scales in this problem. In other cases there are discrepancies between white-noise theory and experiments, such as the photon statistics of a dye laser output where the relative intensity fluctuations tend to increase indefinitely as the laser is weakly excited [12, 13, 14], the reversed asymmetry in the doublet spectrum of double optical resonance is reverted back to normal for lager detuning [15]. Hence, the colored noise model is more realistic than the white-noise model and is widely studied for laser systems, for example in the sideband squeezing in intracavity second-harmonic generation [16], excess quantum noise in a laser [17], and four-wave mixing [18]. In these systems, the colored noise is caused by the laser frequency fluctuation, and the field line shape is Gaussian in some pa-

*Electronic address: xjzhou@pku.edu.cn rameter regime, different with the Lorentzian line shape for white noise.

In this paper we study CARL based on a laser pump with colored noise. Effects of noise on the cooperative scattering of light are studied by stochastic simulation and linear analysis at small correlation time. The Lorentzian bandwidth is related to noise intensity, pump-probe detuning, and the cutoff frequency given by the inverse of correlation time. Effects of these parameters on the growth rate of amplification are analyzed and discussed.

\section{MODEL}

We consider the normal setup for CARL [1, 11, 19]. The backscattered light named probe beam has amplitude $E_{1}$ and frequency $\omega_{1}$, and a partially coherent strong pump field has amplitude $E_{2}$ and the mean frequency $\omega_{2}$. These two beams with almost the same frequency $\left|\omega_{1}-\omega_{2}\right| \ll \omega_{1}$ or $\omega_{2}$, are approximately counterpropagating to form a spatially periodic optical lattice potential, interacting with a very cold and collisionless atomic gas. Atoms moving in this lattice form a density grating, and in turn play a role on the evolution of probe field.

Since $\left|\omega_{2}-\omega_{1}\right|$ is assumed to be very small, the pump or probe wave vector is given by $k=2 \pi / \lambda$ where $\lambda \equiv \lambda_{2} \approx$ $\lambda_{1}$. The pump frequency $\omega \equiv \omega_{2}$ is detuned from the atomic resonance frequency $\omega_{0}$ by $\Delta=\omega-\omega_{0}$. The two-photon recoil frequency is given by $\omega_{r}=2 \hbar k^{2} / m$. In terms of the rescaled time variable $\tau=\omega_{r} \rho t$, the rescaled amplitude of probe field $A=\sqrt{\frac{2 \varepsilon_{0}}{\hbar \hbar \omega \rho}} E_{1}$, the rescaled position $\theta_{j}=2 k z_{j}$ and momentum $p_{j}=\left(m v_{j}\right) /(\hbar k \rho)$ of the jth atom, the modified classical CARL equations with a stochastic pump phase noise are given by [11]

$$
\begin{aligned}
d \theta_{j} / d \tau & =p_{j}, \\
d p_{j} / d \tau & =-A e^{i\left(\theta_{j}-\phi\right)}+c . c ., \\
d A / d \tau & =-\left\langle\left\langle e^{-i\left(\theta_{j}-\phi\right)}\right\rangle\right\rangle+i \delta A, \\
d \phi / d \tau & =\varepsilon(\tau),
\end{aligned}
$$

where the dimensionless CARL parameter is given by $\rho=$ $\left(\frac{\Omega g \sqrt{n}}{2 \Delta \omega_{5}}\right)^{2 / 3}$, the pump Rabi frequency is $\Omega=d E_{2} / \hbar$, the atomic density is $n$, the atom-mode coupling constant is given by 
$g=d \sqrt{\frac{\omega}{2 \varepsilon_{0} \hbar}}$, and the dipole matrix element for the atomic transition is $d$. The average in Eq. (3) is defined as the average over all atoms,

$$
\langle\langle\cdots\rangle\rangle \equiv \frac{1}{N} \times \sum_{j=1}^{N}(\cdots)_{j} .
$$

and the scaled pump-probe detuning is given by $\delta=\left(\omega_{2}-\right.$ $\left.\omega_{1}\right) /\left(\omega_{r} \rho\right)$.

The partial coherence of pump field is described by a phase diffusion model, assumed to evolve according to Eq.(4), where $\varepsilon(\tau)$ is a Gaussian random variable with zero mean $\langle\varepsilon(\tau)\rangle \equiv \overline{\varepsilon(\tau)}=0$, and variance [12, 18, 20]

$$
\left\langle\varepsilon(\tau) \varepsilon\left(\tau^{\prime}\right)\right\rangle \equiv \overline{\varepsilon(\tau) \varepsilon\left(\tau^{\prime}\right)}=\frac{\Gamma}{\tau_{0}} e^{-\frac{\left|\tau-\tau^{\prime}\right|}{\tau_{0}}} .
$$

The colored laser noise given by Eq.(5) is parameterized by the noise correlation time $\tau_{0}$ and the noise strength $\Gamma$, where $\langle\cdots\rangle$ denotes the average over sampling. In the limit $\tau_{0} \rightarrow 0$, from Eq. (5) we get $\left\langle\xi(\tau) \xi\left(\tau^{\prime}\right)\right\rangle \rightarrow 2 \Gamma \delta\left(\tau-\tau^{\prime}\right)$, corresponding to the case of a white noise in laser phase fluctuations with a Lorentzian line shape and linewidth [half width at half maximum (HWHM)] $\Gamma$ for the pump field [11]. When $\tau_{0} \rightarrow \infty$, it recovers the case with a coherent pump laser [1, 11]. For $1 / \tau_{0} \gg \Gamma$, the field line shape is a Lorentzian with a full width at half maximun (FWHM) of $2 \Gamma$, while for $1 / \tau_{0} \ll \Gamma$ it is a Gaussian with a FWHM related to $\sqrt{\Gamma / \tau_{0}}$. The general values of $\Gamma \tau_{0}$ between these two limits lead to Voigt profiles [18], meaning that the laser spectrum is significantly non-Lorentzian for detuning greater than $1 / \tau_{0}$ (from line center) and the spectrum cuts off much more rapidly than a Lorentzian. Consequently $1 / \tau_{0}$ is known as the cut-off [15]. Eq.(5) can also be written as $\dot{\varepsilon}(\tau)+\frac{1}{\tau_{0}} \varepsilon(\tau)=\frac{1}{\tau_{0}} \sqrt{2 \Gamma} \zeta(\tau)$, where $\varsigma(\tau)$ is the Gaussian white noise satisfying $\langle\varsigma(\tau)\rangle=0$, $\left\langle\varsigma(\tau) \varsigma\left(\tau^{\prime}\right)\right\rangle=\delta\left(\tau-\tau^{\prime}\right)$ [16]. As pointed out in the Ref. [11], the amplification due to the partially coherent pump is less sensitive to the atomic distribution in the form of $\exp \left(-\frac{p_{0}^{2}}{2 \sigma^{2}}\right)$ than that due to a coherent pump, where the scaled initial momentum is $p_{0}, \sigma=\frac{\sqrt{3 m \kappa_{B} T}}{\hbar k \rho}, \kappa_{B}$ is Boltzmann's constant, and $T$ is the temperature of atomic gas. In this paper we consider effects of correlation time while assuming $\sigma=0$.

\section{STOCHASTIC SIMULATION}

We directly solve the set of stochastic ordinary differential equations (1)-(4) using stochastic Runge-Kutta algorithms with a colored noise [20]. To conveniently compare with the case of a white noise, in the following we use the same value $\Gamma=5$ and detuning $\delta=2,5,10$, as in Ref. [11]. The scaled probe intensity over time is plotted in Fig 1 to show effects of pump phase diffusion for pump-probe detuning $\delta=2$. The solid line 1 is for the case with white noise $\Gamma=5$ and $\tau_{0}=0$ [11]. The case of coherent pump with $\tau_{0}=0$ and $\Gamma=0$ is shown in the solid line 2 , where the intensity of probe beam is very low and oscillates with time, and the gain

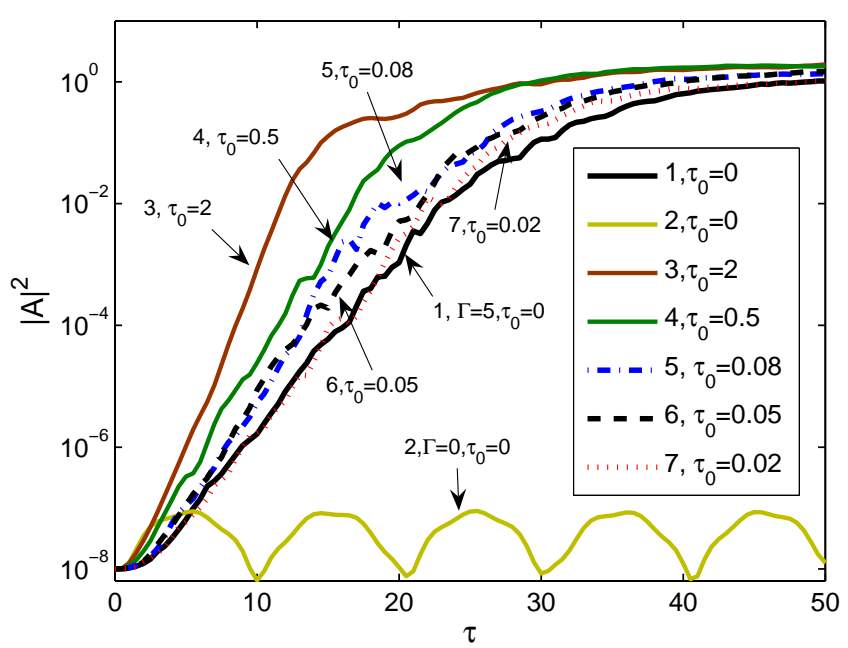

FIG. 1: (Color online) The scaled probe intensity $|A|^{2}$ (averaged over 100 runs) for a cold gas $(\sigma=0)$ by a partially coherent pump with detuning $\delta=2$ for different correlation time $\tau_{0}$. The solid line 2 is for noise intensity $\Gamma=0$, while all other lines are with $\Gamma=5$.

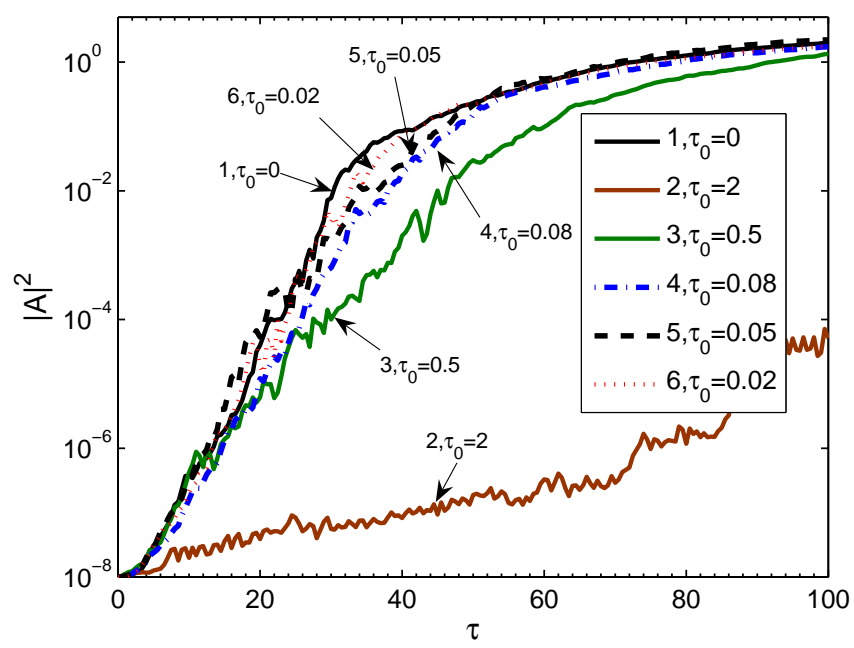

FIG. 2: (Color online) The scaled probe intensity $|A|^{2}$ versus time for detuning $\delta=5, \Gamma=5$.

is suppressed. The growth rate and intensity of the backscattered field greatly increase under the partially coherent pump. In the case of colored noise, the growth rates and amplitudes of the back scattering beams are increased compared to the case of white noise, and this gain increases with correlation time $\tau_{0}$, as shown in the dotted line $\left(\tau_{0}=0.02\right)$, dashed line $\left(\tau_{0}=0.05\right)$, dash-dotted line $\left(\tau_{0}=0.08\right)$, solid line 4 $\left(\tau_{0}=0.5\right)$, and solid line $3\left(\tau_{0}=2\right)$ for $\delta=2$. The correlation time of noise has an opposite effect with $\delta=5$, as shown in Fig.2. The growth rates become slow, and it take more time to get to the saturation than in the case of $\delta=2$. The growth rate and intensity of probe field is suppressed for $\tau_{0}=2$, as shown 


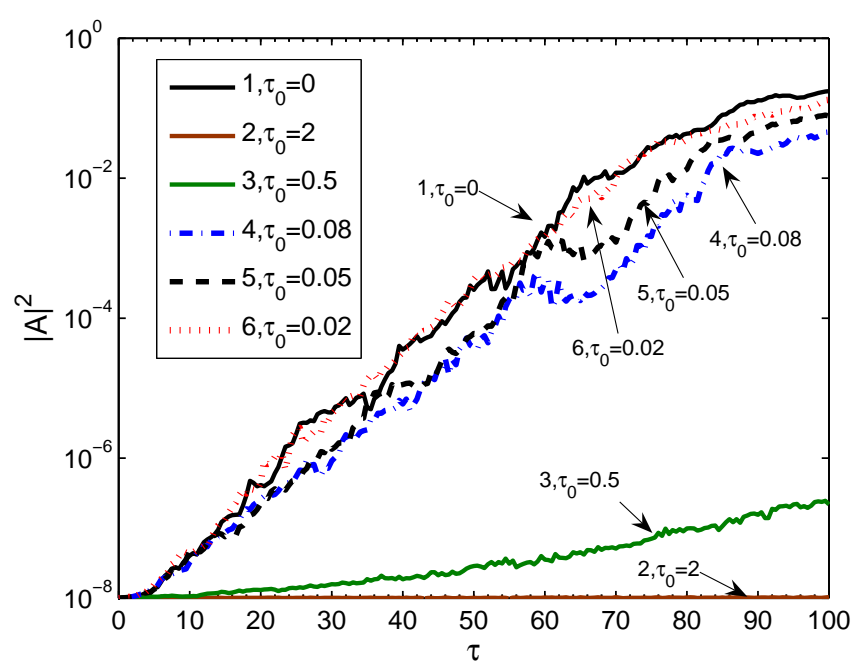

FIG. 3: (Color online) The scaled probe intensity $|A|^{2}$ versus time for detuning $\delta=10, \Gamma=5$.

in the solid line 2. For small correlation time $\tau_{0}=0.02$ (dotted line), it coincides with the case of white noise (the solid line 1 $\left.\tau_{0}=0\right)$. The growth rate decreases with the increase in correlation time, as shown in the dashed line $\left(\tau_{0}=0.05\right)$, and dashdotted line $\left(\tau_{0}=0.08\right)$. However, the difference between the colored noise and white noise at these small correlation times is not so obvious for $\delta=5$. For $\tau_{0}=0.5$, there is a distinct decrease in the growth rate and intensity of probe beam comparing to the case of white noise, as shown in the solid line 3. When the detuning is further increased to $\delta=10$, as shown in Fig 3 the rate and intensity of gain are greatly suppressed for correlation time $\tau_{0}=2$ (solid line 2 ) and $\tau_{0}=0.5$ (solid line 3 ). At small $\tau_{0}$, growth rates also decrease with the increase in correlation time, as shown by the dotted line $\left(\tau_{0}=0.02\right)$, dashed line $\left(\tau_{0}=0.05\right)$ and dash-dotted line $\left(\tau_{0}=0.08\right)$.

The above results show that the gain is suppressed with the increase in correlation time for large detuning $(\delta=5$ and $\delta=10)$. The condition of gain is destroyed with the long correlation time of noise. However for the case with small detuning $\delta=2$, the long correlation time helps to enhance the amplification in the growth rate and amplitude of the probe beam. According to our simulation, there are similar results for $\Gamma=3$ and $\Gamma=1$, where a long correlation time is able to suppress the growth rate for $\delta=5$ and $\delta=10$, and enhance it at $\delta=2$. Thus depending on detuning, the increase in correlation time can either enhance or suppress the gain. To further understand these different behaviors, we carry out the linear analysis at small correlation time in the next section.

\section{LINEAR ANALYSIS}

To analyze effects of colored noise in the regions of instability, we derive the linear evolution equations for the average scattered-field intensity $\overline{A^{*} A}$ from equations Eqs.(1)-(4),

$$
\begin{aligned}
& \frac{d \overline{|A|^{2}}}{d \tau}=\overline{A^{*} b}+c . c ., \\
& \frac{d \overline{A^{*} b}}{d \tau}=-i \overline{A^{*} P}+\overline{b^{*} b}-\left(i \delta+\Gamma^{\prime}\right) \overline{A^{*} b}, \\
& \frac{d \overline{A^{*} P}}{d \tau}=-\overline{|A|^{2}}+\overline{b^{*} P}-\left(i \delta+\Gamma^{\prime}\right) \overline{A^{*} P}, \\
& \frac{d \overline{\left.b\right|^{2}}}{d \tau}=-i \overline{b^{*} P}+c . c . \\
& \frac{\frac{d b^{*} P}{d \tau}}{\frac{d \overline{\left.P\right|^{2}}}{d \tau}}=-\overline{A b^{*}}+i \overline{|P|^{2}}, \\
& \frac{A^{*} P}{c}+c . c .,
\end{aligned}
$$

where $\overline{|A|^{2}}=\overline{A^{*} A}, \overline{|b|^{2}}=\overline{b^{*} b}, \overline{|P|^{2}}=\overline{P^{*} P}$, and

$$
b=\frac{1}{N} \sum_{j}^{N} e^{-i\left(\theta_{j}-\phi\right)}, P=\frac{1}{N} \sum_{j}^{N}\left(p_{j} e^{-i\left(\theta_{j}-\phi\right)}\right) .
$$

To understand how to derive the above formula, here we show the steps in getting Eq. (7). Other equations can be obtained with similar steps. From Eq. (3), we have

$$
\frac{d \overline{A^{*} b}}{d \tau}=-i \overline{A^{*} P}+\overline{b^{*} b}-i \delta \overline{A^{*} b}+i \overline{\varepsilon A^{*} b}
$$

where the last term can be expressed as

$$
\overline{i \varepsilon A^{*} b}=\overline{i \frac{A^{*}}{N} \sum_{j=1}^{N} e^{-i \theta_{j}} e^{i \int_{T}^{\tau_{1}} \varepsilon\left(\tau^{\prime}\right) d \tau^{\prime}} f\left(\tau_{1}, T\right)},
$$

with

$$
f\left(\tau_{1}, T\right) \equiv\left\langle\varepsilon(\tau) e^{\int_{\tau_{1}}^{T} i \varepsilon\left(\tau^{\prime}\right) d \tau^{\prime}}\right\rangle .
$$

We can expand Eq. 15ith the series

$$
\begin{aligned}
f\left(\tau_{1}, T\right) & =\sum_{m=0}^{\infty} \frac{1}{2^{m} m !} \int_{\tau_{1}}^{T} i\left\langle\varepsilon\left(\tau_{\mu}\right) \varepsilon\left(\tau_{1}\right)\right\rangle d \tau_{\mu} \\
& \times\left(\int_{\tau_{1}}^{T} d \tau_{\mu_{1}} \int_{\tau_{1}}^{T} d \tau_{\mu_{2}} i^{2}\left\langle\varepsilon\left(\tau_{\mu_{1}}\right) \varepsilon\left(\tau_{\mu_{2}}\right)\right\rangle\right)^{m} .
\end{aligned}
$$

For small correlation time $\tau_{0}$, it can be further expressed for the moments $m=0$ and $m=1$ only,

$$
f\left(\tau_{1}, T\right) \approx i \Gamma\left(1-e^{-\frac{T-\tau_{1}}{\tau_{0}}}\right)\left[1+\frac{\Gamma \tau_{0}\left(1-e^{-\frac{T-\tau_{1}}{\tau_{0}}}\right)}{2}-\frac{\Gamma\left(T-\tau_{1}\right)}{2}\right] .
$$

Assuming $T=\tau_{1}+K \tau_{0}$ where $K$ is an adjustable parameter, $1 \ll K \ll\left(T-\tau_{1}\right)$, we have

$$
\overline{i \varepsilon A^{*} b}=-\Gamma^{\prime} \overline{A^{*} b}
$$

with

$$
\Gamma^{\prime}=\Gamma\left[1-(K-1) \Gamma \tau_{0} / 2\right] .
$$




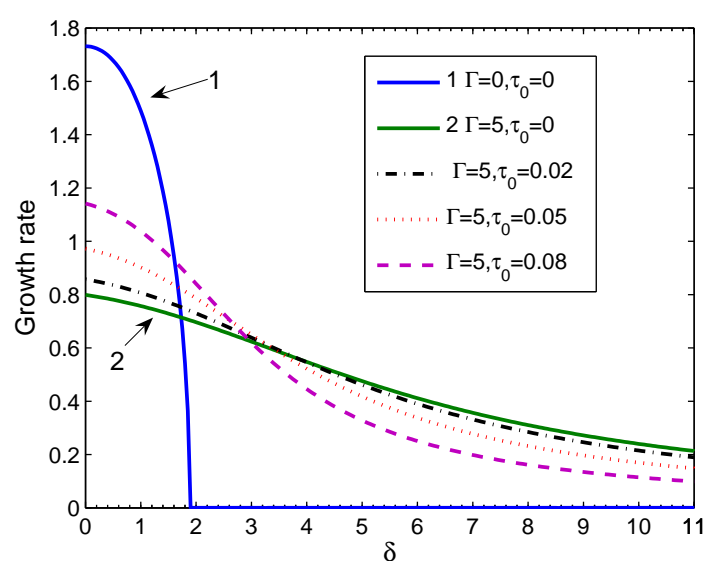

FIG. 4: The growth rate of probe beam $\mathfrak{R} e(\lambda)$ versus detuning $\delta$ for different correlation times. The solid line 1 is for the coherent pump with $\Gamma=0, \tau_{0}=0$, and the solid line 2 is for the partially coherent pump with white noise $\Gamma=5, \tau_{0}=0$. Other lines are for the partially coherent pump with colored noise intensity $\Gamma=5$ and $K=4$, and with correlation time $\tau_{0}=0.08$ (dashed line), $\tau_{0}=0.05$ (dotted line), or $\tau_{0}=0.02$ (dash dotted line ).

Eqs. 611 are identical with equations (5)-(10) in Ref. [11] except that $\Gamma$ for the white noise is now replaced by $\Gamma^{\prime}$ for the colored noise. Thus we can use the relation between the white noise and colored noise models given by Eq.(17) for small $\tau_{0}$. An increase of $\tau_{0}$ can be regarded as decreasing the noise intensity or the linewidth of white noise. Hence, for the initially cold and collisionless atomic gas, we obtain the characteristic root $\lambda$ from the solution of Eqs. 6, 11). The region of instability or amplification satisfies $\mathfrak{R} e(\lambda)>0$. Based on the solution, the growth rates of the probe beams $\mathfrak{R} e(\lambda)$ as the detuning $\delta$ for the different correlation time are plotted in Fig 4

As shown in Fig 4 the maximum growth rate occurs at $\delta=0$. In the case of a coherent pump shown by the solid line 1 , the cutoff is so sharp that no instability or amplification of the probe beam occurs above $\delta_{c}=(27 / 4)^{1 / 3} \approx 1.9$. The noise can extend regions of amplification because the pump phase diffusion broadens the region of instability, as shown by the solid line 2 for the partially coherent pump with a white noise $\Gamma=5, \tau_{0}=0$. The case with small $\tau_{0}=0.02$ is very close to the case of white noise [11]. For detuning $\delta=2$, the growth rates increase with increasing $\tau_{0}$, as shown in Fig.4. However, as the detuning increases, the growth rates decrease with the increase in correlation time for $\delta=5$. Compared to the case of white noise, for example, the growth rate is increased below the critical value $\delta_{0} \approx 3.3$, and decreased above it with the correlation time $\tau_{0}=0.05$, as shown in Fig.4. Hence, depending on detuning, the effect on the growing rate is different for different correlation times under the same noise intensity. For the different noise strength, the growth rates versus detuning with $\tau_{0}=0.05$ are drawn in Fig.5. We got $\delta_{0} \approx 1.8$ for $\Gamma=1$, $\delta_{0} \approx 2.7$ for $\Gamma=3$. The critical detuning $\delta_{0}$ decreases with the decrease in noise strength $\Gamma$. The growth rates are almost

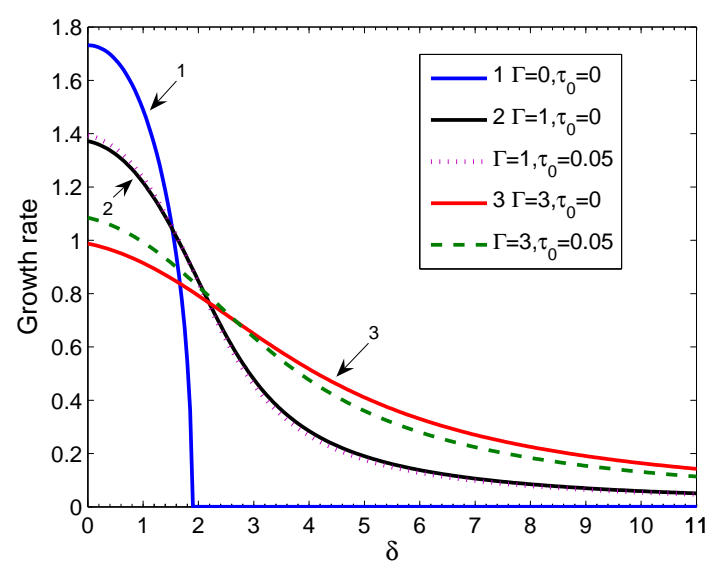

FIG. 5: The growth rate of the probe beam $\mathfrak{R} e(\lambda)$ versus detuning $\delta$ for different noise strength. The solid line 1 is for the coherent pump with $\Gamma=0, \tau_{0}=0$. The solid line 2 is for white noise with $\Gamma=1, \tau_{0}=0$, and dotted line is for colore noise with $\Gamma=1, \tau_{0}=$ 0.05. The solid line 3 is for $\Gamma=3, \tau_{0}=0$, and dashed line is for $\Gamma=3, \tau_{0}=0.05$.

same for white noise and colored noise with small noise intensity $\Gamma=1$, and there is an obvious difference for $\Gamma=3$. The increase in the correlation time is beneficial to the growth rate of the amplification near the threshold detuning, while destructive for the big detuning.

\section{DISCUSSION AND CONCLUSIONS}

The gain of CARL given by the growth rate of the probe beam depends on the frequency of the light field. It has a certain gain bandwidth defined as the spectral range where the light scattering is exponentially amplified. In the amplification process, atom scatter photons from the pump field into the probe beam, experience an acceleration due to the photonic recoil, and occupy different momentum states. On the other hand, the frequency of scattered photons is shifted with respect to the pump-light frequency, resulting in pump-probe detuning. In the case of a noisy pump, there are two velocities involved. One is the stochastic phase velocity with mean value $\left(\omega_{1}-\omega_{2}\right) c /\left(\omega_{1}+\omega_{2}\right)$ of the optical potential, and the other is the atomic velocity. Due to the interaction between the atoms and the optical field, the synchronization between these two velocities eventually leads to the scaled momenta distribution of atoms around a mean value $\langle p\rangle \approx-\delta$, when $\delta>\delta_{c}=1.9$ and the dynamical phase evolution of probe field is negligible. Thus the region of instability extends from the threshold value of the coherent pump $\delta_{c}$ to the bigger detuning [11]. At the same time, a decrease in $\langle p\rangle$ causes an increase in $|A|^{2}$, due to the conservation of momentum $\langle p\rangle+|A|^{2}=$ const. Hence the saturation of instability occurs when the scaled probe intensity satisfies $|A|^{2} \approx \delta$. This is the reason of amplification in large detuning for the partially coherent pump.

However, the noise correlation time $\tau_{0}$, noise intensity $\Gamma$, 
and pump-probe detuning $\delta>\delta_{c}$ greatly affect the growth rate of this amplification behavior. To understand this relation, we define $\gamma$ as the ratio of the effective gain bandwidth $\Gamma_{e f f}$ determined by noise intensity and correlation time to the detuning,

$$
\gamma=\Gamma_{e f f} / \delta
$$

It determines how many momentum states lying within the effective linewidth may participate and be amplified in the CARL dynamics. If this ratio is one, it means that all the momentum states within the linewidth are amplified at the same time and the growth rate is the fastest. If the ratio is more or less than one, this growth rate decreases.

In the case with small correlation times, $\Gamma \ll 1 / \tau_{0}$, the cut-off of the laser spectrum is larger than Lorentzian bandwidth. The colored noise model and white noise model lead to similar results following the relation given by Eq.(17), $\Gamma_{e f f}=\Gamma\left[1-(K-1) \Gamma \tau_{0} / 2\right]$. The effective bandwidth of noise $\Gamma_{\text {eff }}$ limits the range of frequencies accessible for the probe light field. For $\Gamma=5$ and $\delta=2, \gamma \approx 2.5-18.75 \tau_{0}$, the increase in correlation time leads to the decrease in $\gamma$ closer to one. Hence the growth rate of amplification is enhanced with increasing $\tau_{0}$, as shown in Fig.4 of linear analysis and in Fig. 1 of simulation results with $\tau_{0}=0.02,0.05,0.08$. However, for $\delta=5, \gamma \approx 1-7.5 \tau_{0}$, the increase in correlation time leads to a deviation from the match condition, hence increasing $\tau_{0}$ decreases the growth rate, as shown in Fig.4 of the linear analysis and in Fig. 2 of simulation results. Furthermore, for $\delta=10, \gamma \approx 0.5-3.75 \tau_{0}$, increasing correlation time destroys the match condition $\gamma=1$, as demonstrated in Fig.4 of linear analysis and in Fig. 3 of the simulation results with $\tau_{0}=0.02,0.05,0.08$. Based on linear analysis shown in Fig.4, the simulation results at small correlation times shown in Fig.(1-3), and the above analysis, we reach the conclusion that the change in the correlation time can be regarded as adjusting the effective noise intensity. Whether the change in correlation time enhances or suppresses the growing rate depends on whether it helps or destroys the match condition $\gamma=1$.

The large correlation time corresponds to slow frequency fluctuation $1 / \tau_{0} \ll \Gamma$, when the laser shape approaches a Gaussian with the effective bandwidth of HWHM $\Gamma_{\text {eff }}=$ $\sqrt{\left[8(\ln 2) \Gamma / \tau_{0}\right]} / 2$. The effective bandwidth limits the range of frequencies accessible for probe beam. When $\delta=2$, we have $\gamma=1.86,0.93$ for $\tau_{0}=0.5$ and 2. Because 0.93 is closer to unity than 1.86 , the growth rate at $\tau_{0}=2$ is faster than that at $\tau_{0}=0.5$, as shown in the solid line 3 and 4 of Fig.1. However, for $\delta=5, \tau_{0}=0.5,2$ corresponding to $\gamma=0.74,0.37$, the increase of correlation time destroys the match condition and the amplification at $\tau_{0}=2$ is almost suppressed, as shown in the sold line 2 of Fig.2. Furthermore, at $\delta=10$ and $\tau_{0}=0.5,2$, $\gamma=0.37,0.18$, these two values are far from one and the amplification is also suppressed at $\tau_{0}=0.5$, as shown in the solid line 3 of Fig.3. The simulation results show that the growth rate is greatly suppressed with large correlation times. Hence, for big $\tau_{0}$, the increase in correlation time greatly reduces the bandwidth, and suppresses the number of momentum states of participating in the CARL dynamics. The above explanation also is suitable for $\Gamma=3$, while it is not right for weak noise intensity $\Gamma=1$.

It should be possible to observe these effects following the experimental observation of CARL [1, 2] where ultracold ${ }^{87} R b$ atoms were enclosed in a ring cavity. The difference between the experimental setup and our model is that the pump field in the experiment is also in a cavity mode which counterpropagates with respect to the probe beam. The experimental reported characteristic growth time $t_{g}$ for the instability of CARL is about $1 \mu \mathrm{s}$ [6], corresponding to $\omega_{r} \rho \approx t_{g}^{-1}=10^{6} s^{-1}, \rho \approx 10$, and the recoil shift of $\mathrm{Rb}$ as $\omega_{r}=2 \hbar k^{2} / m \approx 2 \pi \times 14 k H z$. For a high finesse of the cavity $\delta \omega_{\text {pump }} \approx 2 \pi \times 20 \mathrm{kHz}$ and the scaled pump linewidth $\Gamma=\delta \omega_{\text {pump }} /\left(\omega_{r} \rho\right) \approx 0.02$, it corresponds to the case of a coherent pump field for $\Gamma \ll 1$. However, the linewidth of the pump field can be adjusted between good-cavity and badcavity regimes by varying the finesse of cavity, atom number, and pump power. $\Gamma$ from 0.3 to 0.2 corresponding to $\rho=4.7$ to 7.0 is the semiclassical good-cavity regime. $\Gamma$ from 3.7 to 2.8 corresponding to the parameter $\rho=5.1$ to 6.7 is the typical semiclassical bad-cavity regime [1, 2]. If we assume $\rho=5$ for $\mathrm{Rb}$, the scaled noise intensity $\Gamma=5$ means a linewidth of the Lorentzian of $2 \pi \times 350 \mathrm{kHz}$, the scaled correlation time $\tau_{0}=0.5$ means the cutoff at frequency $2 \pi \times 140 \mathrm{kHz}$, and pump-probe detuning $\delta=2,5,10$ corresponds to $140 \mathrm{kHz}, 350 \mathrm{kHz}, 700 \mathrm{kHz}$, respectively. Hence, to certain extent, our analysis can be regarded as the CARL in bad-cavity regime which is possible to be accessed in experiments.

In conclusion, the correlation time of pump phase noise greatly affects the growth rate and intensity of cooperative scattering in the system of CARL. The noise makes the amplification region for pump-probe detuning larger than that in the coherent pump. The noise intensity and correlation time determine the effective linewidth of pump laser, not just noise intensity in the case of white noise predicted by phase diffusion model of ideal laser theory. The change in correlation time can enhance or suppress the growing rate depending on the ratio of the effective bandwith to pump-probe detuning which determines how many momentum states within the linewidth are amplified at the same time. This ratio equal to one corresponds to the best match condition for big noise intensity. Whether the growth rate is suppressed or enhanced by the change in correlation time depends on whether it helps or destroy the synchronization condition. These results are useful for analyzing the cooperative scattering process and effects of noise on the collective nonlinear interaction between cold matter and light, and also helpful in studying superradiance from BEC [21] or the phase coherent matter-wave amplification [22] because of the same gain mechanism.

I am grateful to L. Cao and D. J. Wu for their help in the calculation, G. R. M. Robb and X. Xu for their help in the simulation, L. Yin for critical reading our manuscript. I thank two unknown referees for useful and detailed suggestions. This work is partially supported by the state Key Development Program for Basic Research of China (No.2005CB724503, 2006CB921402) and NSFC (No. 60490280). 
[1] S. Slama, S. Bex, G. Krenz, C. Zimmermann, Ph. W. Courteille, Phys. Rev. Lett. 98053603 (2007).

[2] S. Slama, G. Krenz, S. Bex, C. Zimmermann, Ph. W. Courteille, Phys. Rev. A 75063620 (2007).

[3] S. Inouye, A. P. Chikkatur, D. M. Stamper-Kurn, J. Stenger, D. E. Pritchard, and W. Ketterle, Science 285, 571 (1999); D. Schneble, Y. Torii, M. Boyd, E. W. Streed, D. E. Pritchard, and W. Ketterle, Science 300, 475 (2003).

[4] R. Bonifacio and L. De Salvo, Nucl. Instrum. Methods Phys. Res., Sect. A 341, 360 (1994); R. Bonifacio, L. De Salvo, L. M. Narducci, and E. J. DAngelo, Phys. Rev. A 50, 1716 (1994).

[5] G. R. M. Robb, N. Piovella, A. Ferraro, R. Bonifacio, Ph. W. Courteille, C. Zimmermann, Phys. Rev. A 69, 041403 (2004).

[6] D. Kruse, C. V. Cube,C. Zimmermann, Ph. W. Courteille, Phys. Rev. Lett. 91, 183601 (2003).

[7] V. V. Franca, G. A. Prataviera, Phys. Rev. A 75, 043604 (2007).

[8] G. R. M. Robb, B. W. J. McNeil, Phys. Rev. Lett. 94, 023901 (2005).

[9] N. Piovella, M. Cola, R. Bonifacio, Phys. Rev. A 67, 013817 (2003).

[10] N. Piovella, M. Gatelli, R. Bonifacio, Opt. Commun. 194, 167(2001).
[11] G. R. M. Robb and W. J. Firth, Phys. Rev. Lett. 99, 253601 (2007).

[12] S. N. Dixit, P. S. Sahni, Phys. Rev. Lett. 50, 1273 (1983).

[13] R. Short, L. Mandel, R. Roy, Phys. Rev. Lett. 49, 647 (1982).

[14] L. Cao, D. J. Wu, X. L. Luo, Phys. Rev. A 45, 57(1993).

[15] P. Zoller, P. Lambropoulos, J. Phys. B: Atom. Molec. Phys. 12, L547 (1979).

[16] T. A. B. Kennedy, T. B. Anderson, D. F. Walls, Phys. Rev. A 40, 1385 (1989).

[17] A. M. Lee, M. P. Exter, A. L. Mieremet, N. J. van Druten, and J. P. Woerdman Phys. Rev. Lett. 81, 5121 (1998).

[18] G. S. Agarwal, G. Vemuri, C. V. Kunasz, J. Cooper, Phys. Rev. A 46, 5879(1992).

[19] R. Bonifacio, G. R. M. Robb, and B.W. J. McNeil, Phys. Rev. A 56, 912 (1997).

[20] R. L.Honeycutt, Phys. Rev. A 45, 604(1992).

[21] H. Pu, W. P. Zhang, and P. Meystre, Phys. Rev. Lett. 91,150407 (2003).

[22] M. Kozuma, Y. Suzuki, Y. Torii, T. Sugiura, T. Kuga, E. W. Hagley, L. Deng, Science 286, 2309 (1999). 